\title{
Development of the SMA (Speaking Mushroom Approach) Environmental Control System: Automated Cultivation Control System Characterization
}

\author{
Bolaji Oguntoyinbo', Junji HiRama ${ }^{1}$, Hideyuki Yanagibashi², Yoshio Matsui ${ }^{3}$, Toshio Ozawa ${ }^{1}$, \\ Atsushi Kurahashi ${ }^{4}$, Takafumi Shimoda ${ }^{4}$, Shin Taniguchi ${ }^{4}$ and Kouzou NishibOri ${ }^{4}$ \\ ' Kanazawa Institute of Technology, Kanazawa 921-8501, Japan \\ ${ }^{2}$ Kanazawa Technical College, Kanazawa 921-8601, Japan \\ ${ }^{3}$ Kanazawa Gakuin College, Kanazawa 920-1302, Japan \\ ${ }^{4}$ Yukiguni Maitake Co., Ltd., Minamiuonuma 949-6695, Japan
}

(Received March 20, 2014; Accepted July 31, 2014)

\begin{abstract}
This paper describes the principles of automating the Maitake (Grifola frondosa Dicks. Fr.S. F. Gray) mushroom's cultivation process using the developed speaking mushroom approach (SMA) system. The system measures the bioelectric potential signal from the mushrooms and uses it as a control parameter for the lighting conditions. The purpose of the SMA system is to allow optimal control over the cultivation environment in order to improve the running cost and production yields within mushroom factories. It uses sensors and actuators to maintain the optimal temperature and humidity, and uses the known inherent mushroom bioelectric potential to control the lights. The results from the SMA system in this experiment showed a clear bioelectric potential present in the Maitake fruit body. These bioelectric potential signals reflect the internal rhythm of the mushroom as well as external stimulations. Analysing the measured signals the SMA system demonstrated that it will be possible to save energy and time during mushroom cultivation.
\end{abstract}

Keywords : bioelectric potential, cultivation control, morphology, mushroom, SMA

\section{INTRODUCTION}

Ogawa et al. (1997) first conducted research which showed that although mushrooms are not involved in photosynthesis, they exhibit a clear bioelectric potential response to light stimulation. Their research was furthered by Hirama et al. (1997; 2000; 2003) who discovered the greatest bioelectric potential responses were obtained from the 400 to $500 \mathrm{~nm}$ wavelength range (blue LEDs). Also, a correlation was shown between light intensity and measured bioelectric potential (Hirama et al., 2002). While measuring the environmental responses, Yanagibashi et al. (2003) reported that the greatest bioelectric potential was measured at a temperature range of $18^{\circ} \mathrm{C}$ to $22^{\circ} \mathrm{C}$.

The next experiments tested the effects of rhythmic lighting fluctuations on the Maitake fruit body (Yanagibashi et al., 2005). The results suggested that the Maitake fruit body growth is affected by the lighting's on/ off timing. Yanagibashi et al. (2004) revealed that the mushrooms have an internal biorhythm (circadian rhythm), with a period of about 15 to $18 \mathrm{~h}$, which appears in the bioelectric potential signal. They also proved that, even though the total illumination time was only $12 \mathrm{~h}$ light, 12 $\mathrm{h}$ dark (12L12D), $15 \mathrm{~min}$ and $30 \mathrm{~min}$ light timing periods resulted in better morphogenesis than a $24 \mathrm{~L} 0 \mathrm{D}$ cycle.

The initial hypothesis, that the mushroom fruit body produces a bioelectric potential signal that responds to vari- ous environmental changes (Hirama et al., 2008), was then revised to use the fruit body as a bio-sensor for controlling the illumination timing. Yanagibashi et al. (2009) observed that an algorithm to synchronize illumination timing with the Maitake biorhythm, which uses the bioelectric potential rise and fall slope characteristics, can induce a production increase at mushroom farms. Miyazaki et al. (2011) also confirmed that light from blue LEDs increased production yields over other LED colors.

Due to the increase in interest of mushrooms as a health food, the number of mushroom factories has increased over the last few years. Currently, within these mushroom factories, the farmers are using their intuition and experience to decide optimal growth conditions. However, scientific proof of the optimal growth conditions was scarce. Through research, many of the optimal environmental characteristics have been discovered and correlated with the bioelectric potential. This research, from an engineering standpoint, combines the past observations into a Maitake growth test system. We believe it is possible to automatically control the surrounding environment by using the real time bioelectric potential, produced from the mushroom fruit body, as a parameter. This method could increase output yields while saving energy. The system uses the bioelectric potential as a feedback mechanism to adjust the environmental lighting conditions, while maintaining a constant temperature and humidity. The collected research data revealed the mushroom's morphogenesis and

Corresponding author: Bolaji Oguntoyinbo, fax: $+81^{-76}-248^{-5548,}$ e-mail : bolaji@neptune.kanazawa-it.ac.jp 


\section{B. OGUNTOYINBO ET AL.}

bioelectric potential responses to temperature, humidity, $\mathrm{CO}_{2}$, and various lighting conditions.

The latest research further explores the concept of using the Maitake fruit body as a bio-sensor to control lighting. The basis of this new system is a completely automated environmental control system, much like the speaking plant approach (SPA) systems, which have been the subject of many research groups over the years (Hashimoto, 2009). Due to the differences between plant and mushroom physiology, most importantly, the cell structure between the two, many of the traditional methods for controlling the SPA system cannot be used. The title SMA (Speaking Mushroom Approach) is used to describe the particular system upon which this research is based.

\section{MATERIALS AND METHODS}

\section{Bioelectric potential}

Bioelectric potentials exist within mushrooms because of the cell structure. The cell walls are primarily made of lipid molecules and generally do not permit molecules and ions to pass through the barrier (Touyama, 1988). The cell walls have proteins called ion channels that allow only certain types of ions to pass through. Fundamentally, the ion molecules necessary for metabolism are allowed to pass through, while any unnecessary waste is ejected from the cell. Electric potentials are generated as a byproduct of this metabolic reaction (Hiramoto et al., 1994). The channel's open and close conditions control an electrical gradient between the inner and outer cell walls (membrane potential). The membrane potential $E_{m}[\mathrm{~V}]$ is calculated at an ambient temperature of $293 \mathrm{~K}$ using the Nernst equation shown below.

$$
E_{m}=0.058 \log \left(\frac{[C]_{\text {out }}}{[C]_{\text {in }}}\right)
$$

$[C]_{\text {in }}$ and $[C]_{\text {out }}$ are the inner and outer cell ion concentrations. In most plant and animal life, only potassium ion $\left(K^{+}\right)$channels are in a naturally open state and potassium ions can flow with fewer restrictions (Shinmen, 2000). Therefore, any other ion concentrations are considered negligible and the formula, focusing exclusively on $\mathrm{K}^{+}$ion passage changes to:

$$
E_{m}=0.058 \log \left(\frac{[K]_{\text {out }}}{[K]_{\text {in }}}\right)
$$

The bioelectric potential signal is measured by inserting needle electrodes into the fruit body of the mushroom. Between the electrodes exist countless cells, and each respective cell potential contributes to the total transelectrode potential measured. It is believed that the fruit body's physiological and bioelectric response to external stimulations is based on the opening and closing of ion channels in the cell walls (Hirama et al., 2002).

In addition, mushrooms have heliotropism, which is stimulated by blue light and carotenoid or flavonoid photoreceptors. These photoreceptors are thought to create an intermediate reaction that would contribute to the activation of mushroom bioelectric responses through sequential

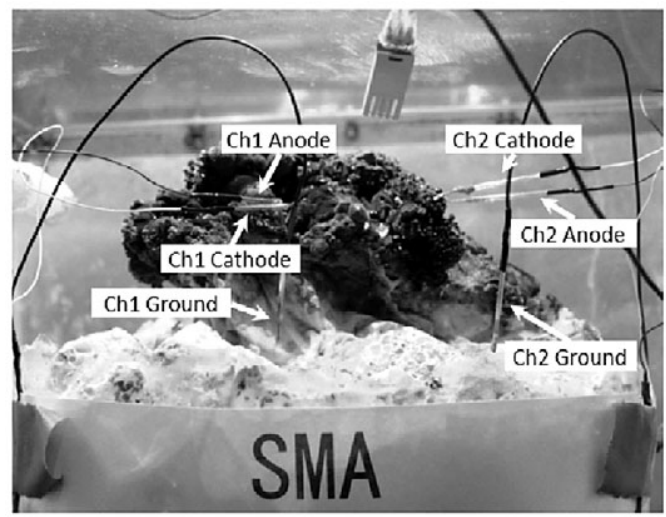

Fig. 1 Bioelectric potential electrode insertion.

chemical reactions (Hirama et al., 2003).

Figure 1 shows a Maitake mushroom with needle electrodes inserted into its fruit body. These induction electrodes were made from a $0.5 \mathrm{~mm}$ chloride coated silver wire. Three electrodes were required to measure a single bioelectric potential signal. The anode and cathode electrodes were inserted into the upper portion of the fruiting body separated by roughly $1 \mathrm{~cm}$. The third electrode, a common or ground wire, was inserted where the base of the fruit body stems from the cultivation bed. The electrodes were connected to a differential amplifier (common-mode rejection ratio: $120 \mathrm{~dB}$ or over, $\mathrm{S} / \mathrm{N}$ ratio: $80 \mathrm{~dB}$ ), specifically built for low noise high gain amplification. Two sets of electrodes were inserted, and are labeled channel 1 (Ch1) and channel 2 (Ch2), because the bioelectric potential behaviour measured from the same mushroom can be irregular. During this experiment, the two sets of needle electrodes were placed in different locations. From these needle electrodes, data used to calculate the four functions explained below was obtained.

SMA system control functions

The environmental parameters for this experiment are shown in Table 1 (a); the function parameters are shown in Table 1 (b). The temperature was held constant at $18^{\circ} \mathrm{C}$ throughout the growth period. The relative humidity alternated between $90 \%$ and $95 \%$ RH every $30 \mathrm{~min}$. Within this humidity range, the mushrooms produced the highest bioelectric potential response. The highly sensitive bioelectric potential response to humidity was already verified (Ozawa, 2012). When the mushroom's response to the humidity was strong, the mushroom physiological activity was also strong. When the response to humidity was weak, the mushroom growth activity was also found to be weak. The SMA system utilized this correlation to help control the environmental lighting. The flowchart of Fig. 2 shows the general system procedure. The system measured the bioelectric potential in real time. The results were sampled into four different functions. They were root means square (RMS), slope, long term fast Fourier transformation (FFT), and short term FFT. These four functions were then used to set the lighting conditions.

This experiment also included an algorithm which measured the bioelectric potential from both channels and 
Table 1 SMA system parameters.

(a) Environmental parameters

\begin{tabular}{ccccc}
\hline System Data Category & \multicolumn{4}{c}{ SMA System Test Variables } \\
\hline Environmental Conditions & Temp $\left[{ }^{\circ} \mathrm{C}\right]$ & $\begin{array}{c}\text { Relative Humidity } \\
\text { High }[\% \text { RH }] \\
\text { Relative Humidity } \\
\text { Low }[\% \text { RH }] \\
90 \pm 5\end{array}$ & $\begin{array}{c}\text { Humidity Toggle Time } \\
{[\text { Seconds }]} \\
1800\end{array}$ \\
\hline \multicolumn{5}{c}{ (b) Function parameters } \\
\hline Function Parameters \\
Threshold & $18 \pm 1$ & RMS & Long Term FFT & Short Term FFT \\
Window Size* $[$ Points $]=[\mathrm{min}]$ & $0.6[\mathrm{mV} / 30 \mathrm{~min}]$ & $0.025[\mathrm{~V}]$ & $17.5[\mathrm{~dB}]$ & 0.12 [arb] \\
Running Window Shift* $[$ Points $]=[\mathrm{min}]$ & 30 & 3000 & 2048 & 256 \\
\hline
\end{tabular}

*1 point equals 1 min

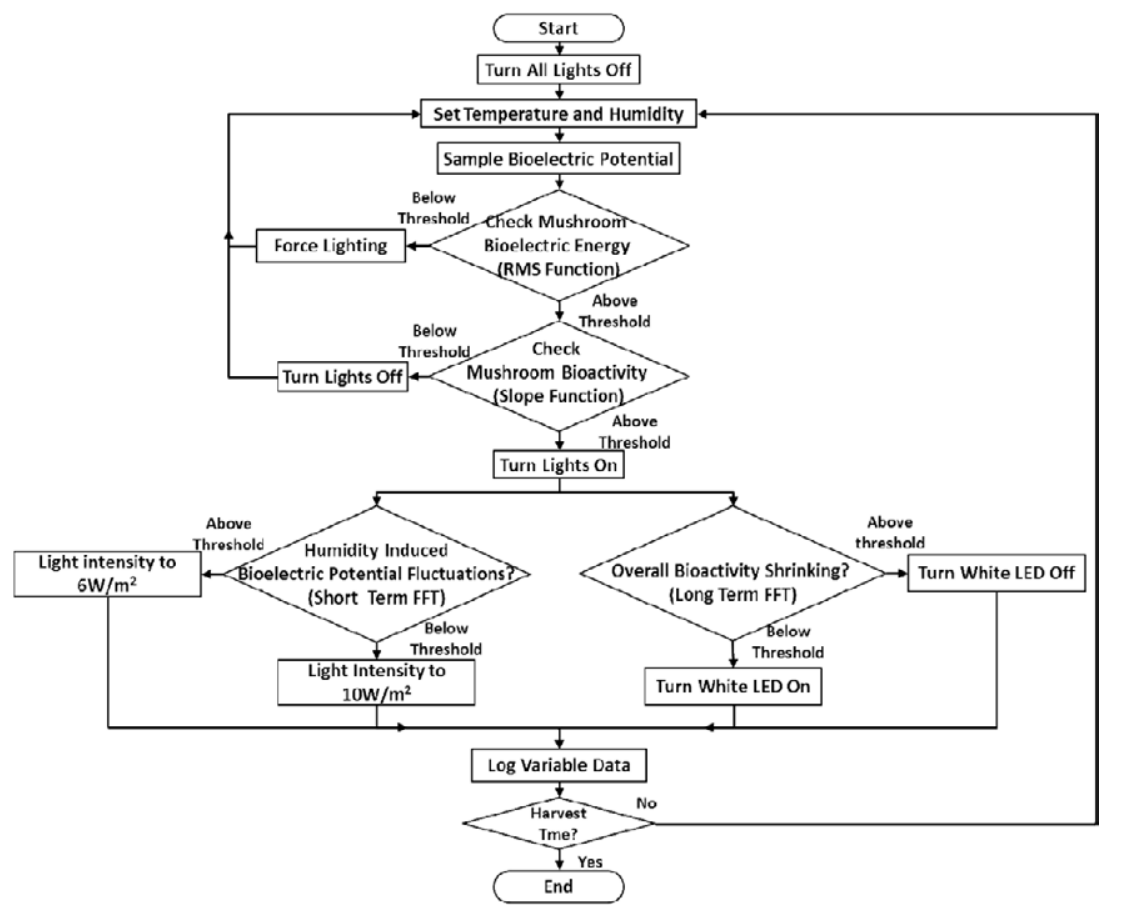

Fig. 2 SMA control flowchart.

sampled them into the four functions. This enabled the SMA system to select the measured bioelectric potential from either channel. The channel data used to calculate each of the four functions could then be used at any time to control lighting conditions. Furthermore, the four functions used for the SMA system lighting control were managed through an OR operation to avoid prioritizing.

The mushroom bioelectric potential is sensitive to external ambient environmental changes. The RMS calculation, the standard deviation calculated from the measured bioelectric potential value in Volts (V), was used to measure and evaluate this characteristic. Within the specified analysis window, the trend component (DC component) was removed and only the AC component was calculated. When the RMS voltage was substantial, the mushroom's response to its external environment was apparent and active growth was expected. When the RMS voltage was small, the growth activity was considered to be weak. As a result, the strongest light $\left(10 \mathrm{~W} \mathrm{~m}^{-2}\right)$ intensity was applied. The RMS function controlled the overall system lighting and was used to stimulate the early stages of growth, and to prevent weak growth throughout the entire cultivation period. The RMS had a measurement window of $50 \mathrm{~h}$; every $12 \mathrm{~h}$, the measurement was shifted and recalculated. The RMS threshold was selected through the testing of numerous conditions and through trial and error (Yanagibashi et al., 2009).

The slope function, the rise and fall rate to turn the lighting on or off, was used to decide how quickly the trend component of the bioelectric potential rises and/or falls within a specific time interval. When the slope dropped below the threshold, the mushroom was considered to be in a rest state and the lights were turned off. When the slope rose above the threshold, the mushroom was considered to be in an active state and the lights were turned on. The slope was calculated by averaging the measured bioelectric potential within a $30 \mathrm{~min}$ analysis window. After the initial calculation, the running window was shifted at one min intervals and recalculated. Each time the running window was calculated, the resulting value was compared to the threshold. The slope threshold value was selected from the results of previous experiments (Hirama et al., 2008). By selecting the threshold from the slope rise and fall characteristics, the lighting on/off conditions were controlled. 
The slope function was used to promote mushroom growth while reducing light source power consumption.

The short term FFT calculation was used to determine the level of activity from the bioelectric potential. When the mushroom's growth was sufficiently active, the bioelectric potential response sensitivity was high enough to follow the changes in humidity. Table 1 shows the humidity conditions. The humidity within the SMA system was set to fluctuate from $90 \% \mathrm{RH}$ (relative humidity) to $95 \% \mathrm{RH}$ every thirty min. The analysis window of 256 min was shifted every $60 \mathrm{~min}$ and was recalculated to capture the strength of the 60 min bioelectric potential periodic component. The threshold was set to estimate growth activity and then control the light source intensity as shown in Fig. 2. The short term FFT was targeted at effectively promoting growth at all times by applying a strong light intensity only when necessary.

The long term FFT is used to signal the end of the mushroom growth period. The mushroom bioelectric potential demonstrates a circadian rhythm (roughly 0.5 to 1 day fluctuation period). This fluctuation period was used by the long term FFT to calculate the mushroom's overall growth activity. The long term FFT analysis window of about 1.4 days was shifted every $30 \mathrm{~min}$ and recalculated. If the calculated FFT value was small enough, blue and white LEDs were both activated to stimulate mushroom growth. Also, when the harvest time was near, blue and white LEDs were turned on to promote as much growth as possible in the final stages. During most of the test, the 10 $\mathrm{W} \mathrm{m}{ }^{-2}$ blue LED intensity alone was sufficient for proper mushroom morphogenesis. When the circadian rhythm became weak, it was considered that the intensity from blue LEDs were inadequate. Therefore, the white LED was included to aid stimulation of the mushroom bioactivity. The white LED was chosen as an energy efficient replacement for the fluorescent lighting (normally used in mushroom factories).

Mushroom factory model: overview

Figure 3 shows the proposed SMA system factory model. The system depended on the following conditions to operate: light wavelength, light intensity, temperature, humidity, and the data logging function. The system used both blue and white LED colors to stimulate mushroom growth. The light intensity was increased or decreased to save energy whenever possible. The temperature and humidity ranges were constantly maintained at $18^{\circ} \mathrm{C}$ and $90 \%$ $\mathrm{RH}$ to $95 \% \mathrm{RH}$ respectively. The data logging function measured and recorded all the parameters listed above, along with the bioelectric potentials. The proposed factory system relied on the bioelectric potential from a single master mushroom used to manipulate the lighting for all the cultivating mushrooms. In this study, the SMA system that was constructed for laboratory use tested the factory model operation on a single mushroom at a time.

SMA system overview

Figure 4 shows the test chamber used to conduct the SMA automated experiments. The system consisted primarily of an outer shield box, an environmental control unit, and an inner acrylic enclosure. The mushroom was

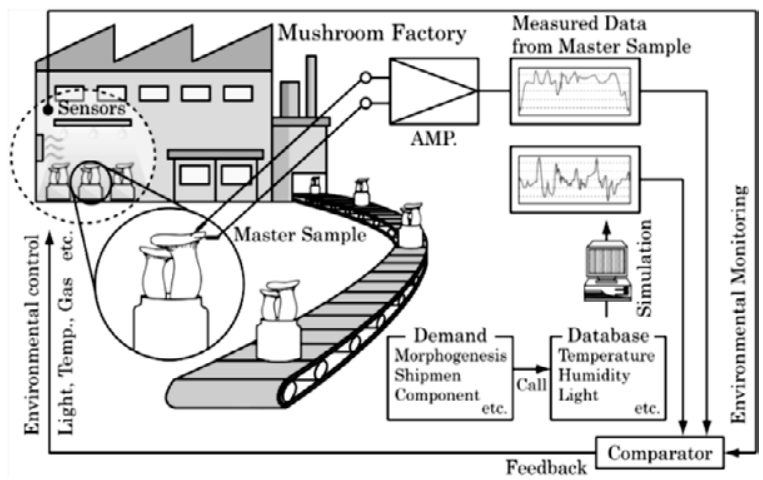

Fig. 3 SMA system factory model.

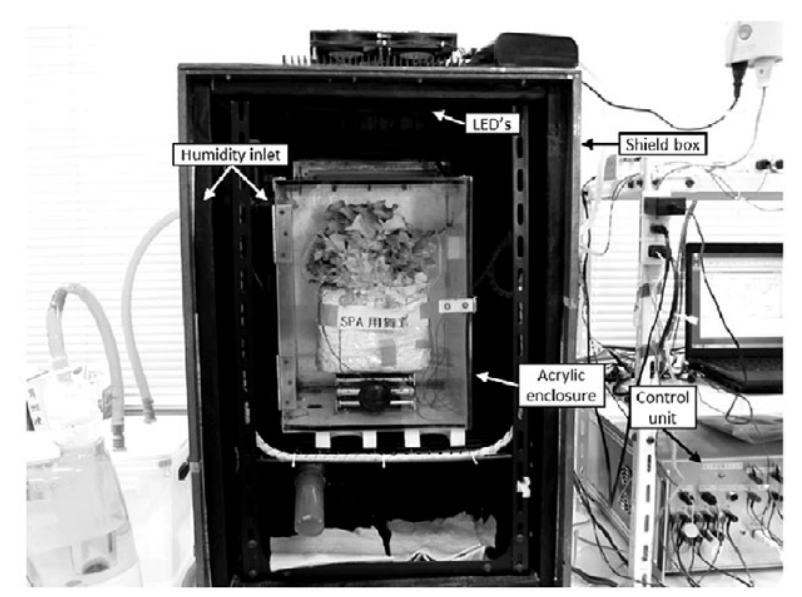

Fig. 4 SMA Environmental chamber, internal view. The mushroom is cultivated inside acrylic enclosure. One temperature and one humidity sensor is placed in the acrylic enclosure. The second temperature sensor is placed just outside of the acrylic enclosure. The SMA system constantly monitors both sets of sensors, and closely controls the environment within the acrylic enclosure. The control unit (partially shown) is responsible for taking the sensor information and sending it to the PC.

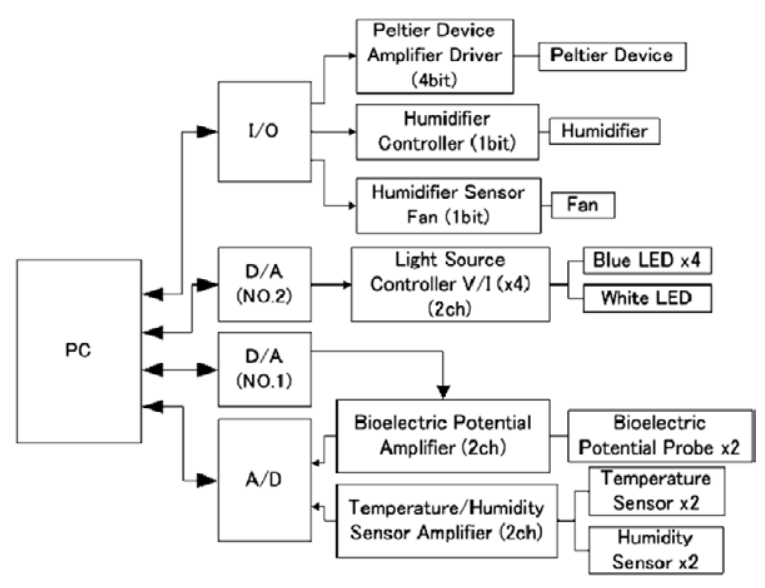

Fig. 5 Control system hardware layout. The SMA system utilizes two sets of temperature and humidity sensors. The first temperature and humidity set is used to monitor the mushroom growth environment inside the acrylic enclosure. The second set is used to monitor the environment outside of the acrylic enclosure but inside the shield box. 
placed inside the inner acrylic enclosure, during the fruit body's fifth day primordial state on the fungus bed. The LEDs were mounted on the inner top of the shield box and also attached to a heat sink extruding from the top of the outer chamber. The system used four blue $(455-470 \mathrm{~nm})$ $10 \mathrm{~W}$ LEDs and one separately controlled white $20 \mathrm{~W}$ LED. The peltier cooler (not shown) was attached so that it extended from the back of the shield box. The environmental control unit (partially shown) was responsible for controlling and measuring the environmental conditions and bioelectric potential feedback. This control unit was attached to a PC via USB, and the data was sent to a PC in real time while the experiments were under way. All measured information was recorded on the PC.

SMA system hardware overview

Figure 5 shows the basic hardware diagram used for this system. The digital I/O, the digital to analog converters (DAC), and the analog to digital converters (ADC) were used to control or measure all the devices used by the sys- tem. The digital I/O controlled the peltier device, humidifier, and humidifier sensor fan, while the DAC controlled the bioelectric potential amplifier bias and color and intensity of the system lighting. The ADC was used to measure the bioelectric potential feedback from the amplifier and the chamber's internal temperature/humidity sensors.

\section{RESULTS AND DISCUSSIONS}

Figure 6 shows the channel 1 and 2 bioelectric potential responses, compared to the temperature, humidity, and light conditions from the experiment. Throughout the 14 day test, a similar circadian rhythm was recognized from the measured bioelectric potential on both sensor channels. Even though the two signals were not in perfect phase, the same overall trend was present. The Maitake in this particular test had high bioelectric potential levels. The location of needle electrode insertion was a possible reason for the potential increase and therefore the details should be

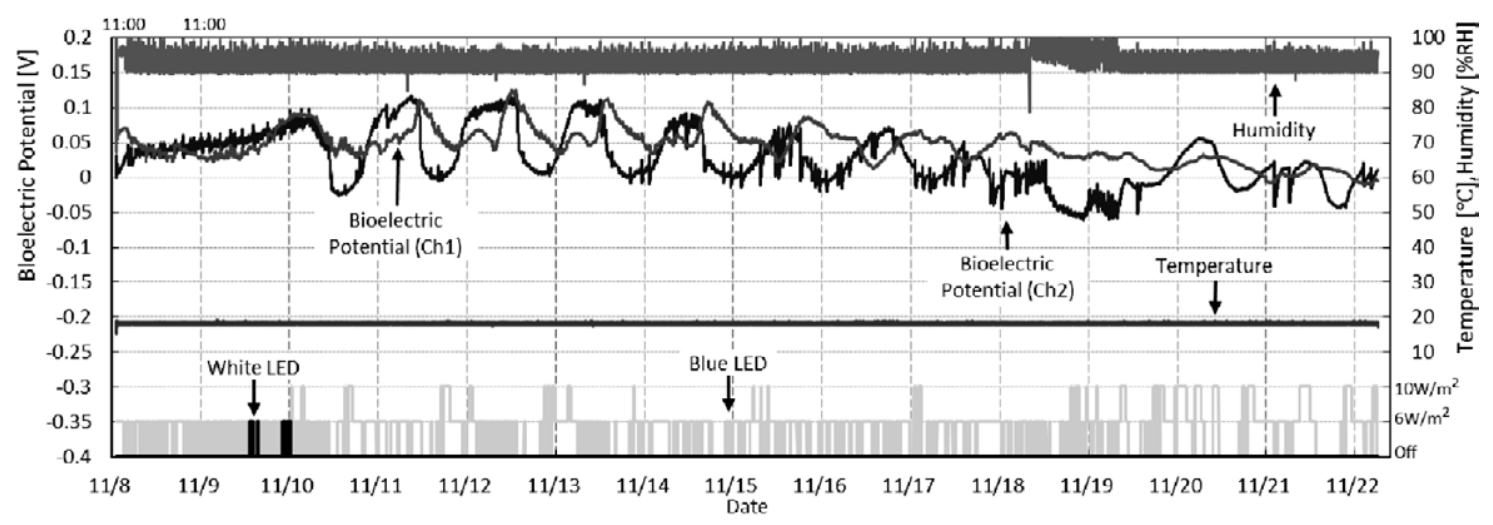

Fig. 6 Measured test results.

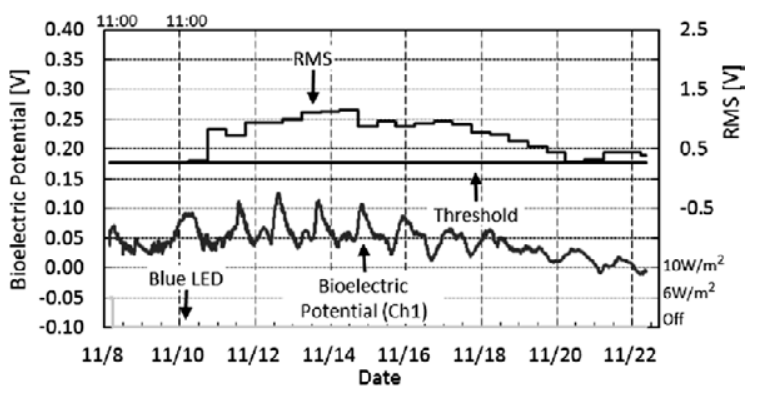

(a) Channel 1

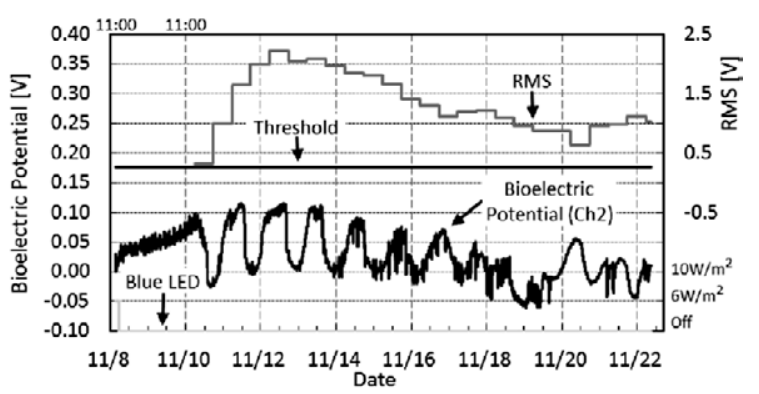

(b) Channel 2

Fig. 7 (a) Channel 1 RMS. (b) Channel 2 RMS.

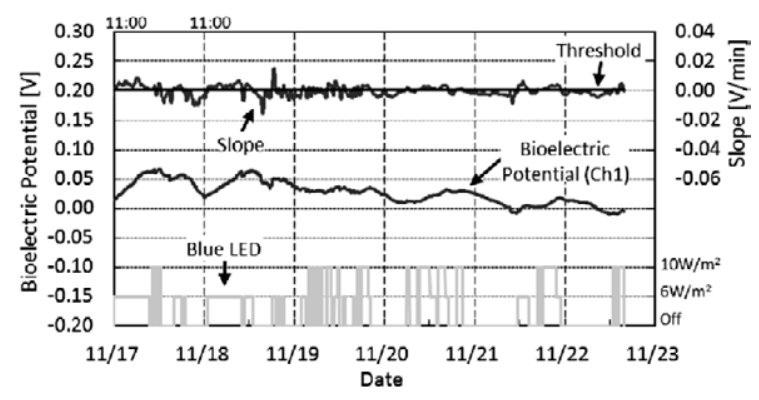

(a) Channel 1

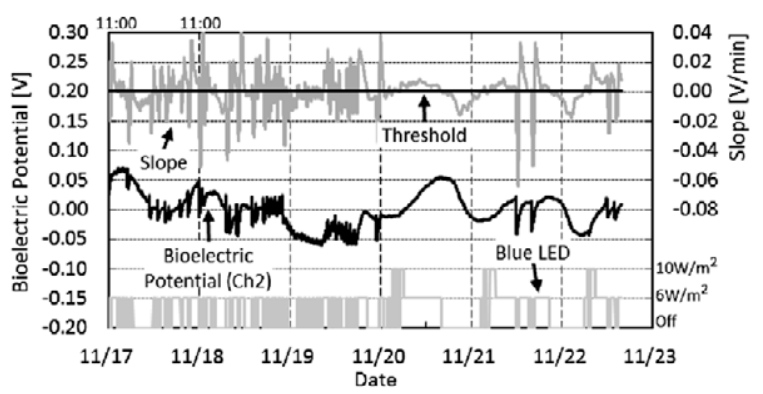

(b) Channel 2

Fig. 8 (a) Channel 1 slope. (b) Channel 2 slope. 
examined further.

Biorhythm control: RMS

Figure 7(a) and (b) show the channel 1 and channel 2 respective RMS results. The calculated RMS, RMS threshold, measured bioelectric potential, and light conditions are shown. The mushroom had strong vital signs therefore the remaining lighting functions gained priority. The calculated RMS from either channel did not drop below the threshold and the RMS trends were similar. This suggests that wherever the electrodes are placed, the resulting feedback will always have similar properties. However, there was a difference in the measured bioelectric potential signals. This could also be related to the electrode location in relation to the environmental stimulations, such as lighting source or humidity inlet (Fig. 3).

Biorhythm control: slope

Figure 8(a) and (b) show the slope results of both channels from the last six days of the experiment. Channel 1 showed a cleaner bioelectric potential signal, thus the calculated slope was present, yet less dramatic. On the other hand, the sharp fluctuations shown in the channel 2 bioelectric potential signal greatly affected the calculated slope activity. It is possible that the sharp changes within the channel 2 bioelectric potential signal could be attributed to the fluctuations in humidity. These channel 2 humidity induced fluctuations would have caused the calculated slope to swing as much as 0.08 [ $\left.\mathrm{V} \mathrm{min}{ }^{-1}\right]$, unintentionally triggering the lighting on/off control. However, the channel 1 calculated slope was as intended and was not affected by the humidity induced fluctuations. As a result, the overall light timing for channel 2 was increased over channel 1. The one day average light timing for channel 2 and channel

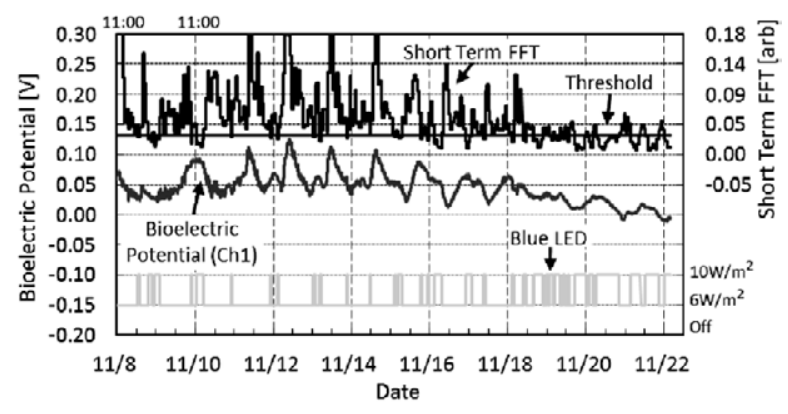

(a) Channel 1

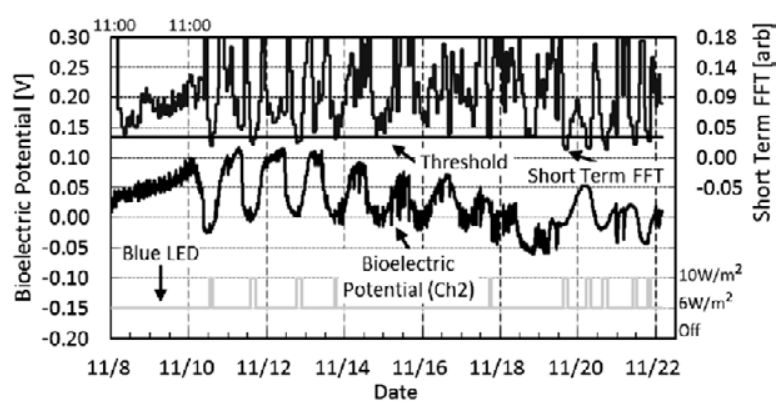

(b) Channel 2

Fig. 9 (a) Channel 1 short term FFT. (b) Channel 2 short term FFT.
1 was $12.7 \mathrm{~h}$ and $9.5 \mathrm{~h}$ respectively.

The results from this experiment show that when measured potentials are large, good morphogenesis can be expected. However, factors such as probe insertion location can cause variances in measured potentials. We believe that, through further research and by implementing well-adjusted thresholds, these potential variances can be compensated for.

\section{Biorhythm control: short term FFT}

Figure 9(a) and (b) show the calculated short term FFT from channel 1 and channel 2 for this experiment. When the calculated value dropped below the threshold, the lighting intensity increased from $6 \mathrm{~W} \mathrm{~m}^{-2}$ to $10 \mathrm{~W} \mathrm{~m}^{-2}$. When the calculated short term FFT was above the threshold, the bioelectric potential clearly followed the change in humidity, and the mushroom's physiological properties became more active. Throughout the test, the weaker channel 1 produced insufficient bioactivity in response to the humidity fluctuations, thus triggering an intensity increase roughly once or twice a day for the first eight days. During the last six days of the growth cycle, the calculated channel 1 short term FFT often caused the light intensity to increase to $10 \mathrm{~W} \mathrm{~m}^{-2}$. This coincided with a weaker measured bioelectric potential, and was furthered by a progressively weakening measured potential from the last six test days. The channel 2 test results also exhibited some intensity increases, but the applied $10 \mathrm{~W} \mathrm{~m}^{-2}$ occurred only about once a day and with increasing frequency in the last three days. Upon further inspection, the intensity increase seemed to occur regularly at the trough in the circadian rhythm for both signals and occasionally at other locations. The actual circadian rhythm was not calculated in the short term FFT due to its $256 \mathrm{~min}$ running window. Also, the light inten-

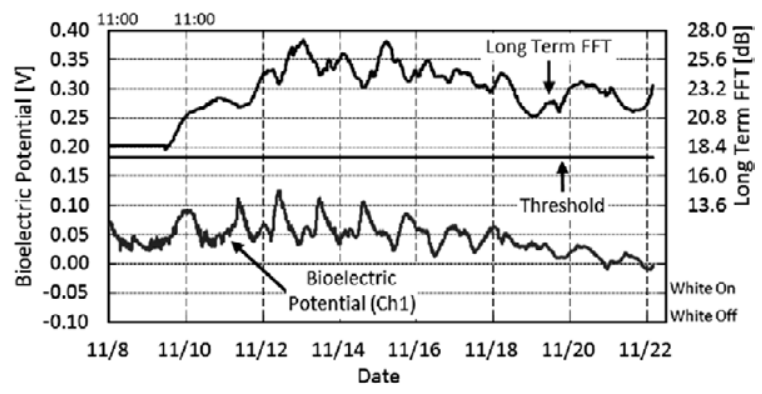

(a) Channel 1

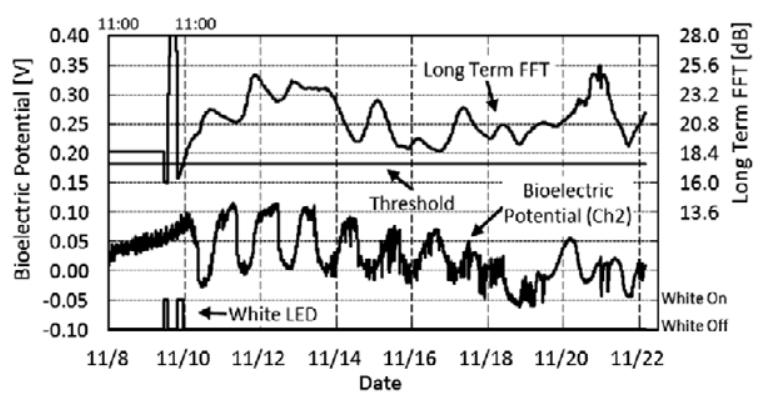

(b) Channel 2

Fig. 10 (a) Channel 1 long term FFT. (b) Channel 2 long term FFT. 


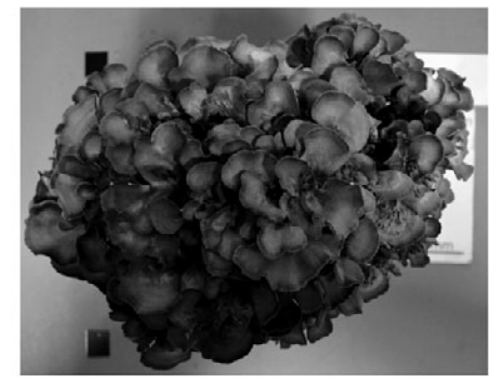

(a) Top view

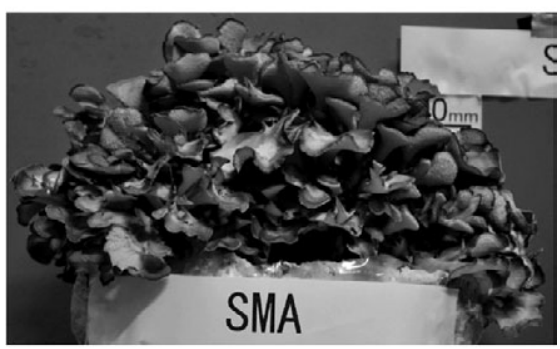

(b) Front view

Fig. 11 (a) Maitake fruit body (Top). (b) Maitake fruit body (Side).

sity changes in Fig. 9 were the result of all four functions combined and the short term FFT lighting control results could not be separated.

Biorhythm control: long term FFT

Figure 10(a) and (b) show the calculated long term FFT from channels 1 and 2 respectively. The white LED briefly turned on when the first long term FFT calculation was completed. This was caused by channel 2's linear bioelectric potential signal from the first two test days. The calculated FFT of the linear signal produced unexpected values for that time period. The unexpected channel 2 results were considered anomalies. On the other hand, the channel 1 bioelectric potential generated a circadian rhythm almost immediately. The difference in the two measured potentials was associated with the insertion location of the needle electrodes in the mushroom. At the end of the test, the white LED was not triggered because the Maitake produced a strong circadian rhythm from both channels. Furthermore, the $30 \mathrm{~min}$ humidity induced fluctuations did not affect the long term FFT calculations. The larger 1.4 day window captured the overall circadian rhythm of the mushroom and the humidity responses were too small to be calculated.

\section{Morphogenetic properties}

Figure 11 shows the resulting Maitake mushroom from the top and front. The resulting fruit bodies were healthy and evenly dispersed. The coloration of the mushroom was also considered to be normal. No defects or abnormalities were present. The fruit body weight at harvest was $1040 \mathrm{~g}$, which falls within an acceptable range for mushroom farm yields.

Power consumption

Upon comparison of the overall lighting with the 12L12D timing found in mushroom factories, the SMA system had an overall average lighting time of $14.1 \mathrm{~h}$, an increase of just over two hours. We believe that through system improvements, an increase in efficiency is possible. The next objective is to better control the lighting conditions. The overall light timing was increased over the standard 12L12D, but with further system and threshold development an efficiency increase is expected.

This experiment was conducted three times. The power consumption outcome and the other obtained data explained above were similar for each iteration. Using these research results, the system will be tested for growth promoting effects, and running cost reduction, both in a laboratory and factory locations.

\section{CONCLUSION}

In this experiment, the theory of using the mushroom's bioelectric potential as a basis for an environmental control system was tested. The mushroom itself was used as a biosensor to operate four lighting conditions. The SMA system's operation in the laboratory stage was confirmed. The resulting mushroom morphogenesis, size, and weight were equivalent to typical yields found in mushroom factories. Development will continue so that the SMA system's light timing becomes comparable or better than the standard 12L12D currently found in factories. We believe that it is possible to use the SMA system to maximize mushroom factory productivity while decreasing overall energy usage and production running costs. The SMA laboratory system was constructed as a method to test the theory of the SMA system factory model. Upon completion of the laboratory system, the system will be implemented in a factory setting and its operations validated.

\section{REFERENCES}

Furukawa, H. 1992. Mycology. (in Japanese) Kyoritsu Publishing, Tokyo, pp 1.

Hashimoto, Y. 2009. How to approach environmental control in agriculture. Its history, SPA and the future of the greenhouse horticulture. (in Japanese with English summary) J. SHITA 21: $2^{-6}$.

Hirama, J., Matsuoka, D., Matsui, M., Nishibori, K. 2008. Mushroom farming using illumination control by synchronization with spontaneous rhythm of the bio-electric potential of Grifola frondosa (Maitake Mushroom). (in Japanese) J. SHITA 20: 90-97.

Hirama, J., Matsuda, M., Yanagibashi, H., Miyamoto, T. 2003. Relationship between the bio-electric potential and the morphogenetic properties of Grifola frondosa when subject to different light stimuli. First Japan-Thailand Symposium on Advanced Technologies in Agricultural Production and PostProduction Operations, December, Chang Mai, Proceedings $\mathrm{p}$ 89-94.

Hirama, J., Miyamoto, T., Hiroaki, S., Takahashi, N., Nishibori K., Ohdaira, Y. 2000. Effect of a specific light quality on the morphogenetic properties for grifora frondosa. Part 1. comparison of irradiation methods (continuous or $1 / \mathrm{f}$ fluctuating light stimuli) and characteristics of light wave length. (in Japanese with English summary) J. SHITA 12: 190-194.

Hirama, J., Takahashi, N., Miyamoto, T., Miura, H., Tazawa, S. 1997. Bio-electric potential under light stimulation of grifora frondosa. Part 2. (in Japanese with English summary) J. 


\section{B. OGUNTOYINBO ET AL.}

SHITA 9: 278-281.

Hirama, J., Yanagibashi, H., Matsuda, M., Ishida, R., Miyamoto, T. 2002. relationship between the bio-electric potential and morphogenetic properties of Grifola frondosa under different light stimuli. (in Japanese with English summary) Trans. IEE Jpn. 122-C: $1679-1685$.

Hiramoto, A., Moori, H. 1994. Introduction to biology. (in Japanese) Foundation for the Promotion of the Open University of Japnn, Wakaba, p 39-47.

Miyazaki, Y., Masuno, K., Abe, M., Nishizawa, H., Matsumoto, T., Kunitomo, S., Sakata, H., Nakamura, K., Koyama, T., Ito, M., Kazama, H., Suzuki, D., Obatake, Y., Sano, H., Nakamura,M., Miyazaki, K., Sakamoto, Y., Kaneko, S., Kamada, T. 2011. Light-simulative effects on the cultivation of edible mushrooms by using blue LED. ICMBMP7, Arcachon, October, Proceedings Vol. 2, p 58-67.

Ogawa, T., Miura, H., Hirama, J., Miyamoto, T., Hirota, N. 1997. Bio-electric potentials for the light stimulation of Maitake mushroom. Part 1. (in Japanese) J. SHITA 9: 89-92.

Ozawa, T., Oguntoyinbo, B., Kawabata, K., Hirama, J., Yanagibashi, H., Matsui, Y., Kurahashi, A., Shimoda, T., Taniguchi, S., Nishibori, K. 2012. SMA (Speaking Mushroom Approach) environmental control system development based on mushroom bio-electric potential: prototype performance test. (in Japanese) JSABEES 50th Anniversary
Memorial Meeting, September, Tokyo, Proceedings C33: 5051.

Shinmen, T. 2000. Illustrated Cell Mechanisms. (in Japanese) Natsume Publishing, Tokyo, p 102-103.

Touyama, S. 1988. Introduction to cellular biology. (in Japanese) HBJ Publishing, Tokyo, p 53-66.

Yanagibashi, H., Matsuda, M., Harada, K., Hirama, J., Miyamoto, T., Nishibori K., Ohdaira, Y. 2003. Bio-electric potential under light stimulation of Grifola frondosa. Part 3. Relationship between the environmental temperature and the bio-electric potential. (in Japanese) J. SHITA 15: 94-97.

Yanagibashi, H., Matsuda, M., Hirama, J., Miyamoto, T., Nishibori K., Ohdaira, Y. 2004. Bio-electric potential under light stimulation of Grifola frondosa. Part 4. Characteristics of rhythmic fluctuation of the fruit body and the primordium. (in Japanese) J. SHITA 16: 193-197.

Yanagibashi, H., Hirama, J., Matsuda, M., Miyamoto, T. 2005. Investigation of the optimum farming temperature for Grifola frondosa and growth promotion using the bio-electric potential as an index. IEEJ Trans. SM. 125: 159-164.

Yanagibashi, H., Hirama, J., Nobuyuki, N., Imazawa, A. 2009. Mushroom farming using illumination control by synchronization with the bio-electric potential of Grifola frondosa. ASET, Dalian, China, October, Proceedings S5-7. 